\section{P09 THE USE OF COCHRANE REVIEWS IN CLINICAL GUIDELINES FOR RESPIRATORY DISEASE IN CHILDREN IN THE UNITED KINGDOM - A SYSTEMATIC REVIEW}

\author{
doi:10.1136/archdischild-2013-304107.009
}

${ }^{1}$ THN Cox, 'AR Smyth, 'AP Prayle, ${ }^{2} \mathrm{DA}$ Hughes, ${ }^{3} \mathrm{~J}$ Rycroft-Malone, ${ }^{4} \mathrm{~K}$ Thomas. ${ }^{1} \mathrm{Child}$ Health, University of Nottingham, Nottingham, UK; ${ }^{2}$ Centre for Health Economics \& Medicines Evaluation, Bangor University, Bangor, UK; ${ }^{3}$ Health Services \& Implementation Research, Bangor University, Bangor, UK; ${ }^{4}$ Centre of Evidence Based Dermatology, University of Nottingham, Nottingham, UK be used for guideline recommendations. We assessed the use of CRs in UK guidelines for lower respiratory disease in children and the agreement between the guideline recommendations and the CRs.

Methods We searched Embase, Pubmed and the websites of guideline commissioning agencies for clinical guidelines. For each guideline recommendation, we identified relevant CRs in the Cochrane Library. We noted whether the CRs were cited in the guidelines and if they agreed with the guideline recommendations. Two investigators independently assessed CRs for relevance and agreement. We investigated the influence of the guideline commissioning agency and the topic, upon whether CRs were cited and whether their conclusions were followed. We investigated factors influencing the use of CRs, using logistic regression.

Results We identified 21 guidelines which made 1025 recommendations, of which 555 were recommendations for treatment of lower respiratory disease in children. For 115 of these 555 recommendations $(21 \%)$ we identified a CR which could inform the recommendation. Approximately, one third of these recommendations (40/115) did not use any of the available CRs or used only some. The guideline commissioner had a significant influence on the use of CRs ( $p=0.03$ ), with BTS guidelines performing best. Guidelines on some topics eg cystic fibrosis were significantly more likely to cite CRs than others eg asthma ( $p=0.007)$. In 20/115 guideline recommendations there was not full agreement with the CR Conclusion $9(8 \%)$ disagreed, $6(5 \%)$ partial agreement and $5(4 \%)$ the guideline made a strong recommendation not supported by the CR.

Conclusion In spite of the work of the Cochrane collaboration, there are still many treatment decisions where there is no systematic review to inform guideline recommendations. However, we have shown that, even where a CR exists, guideline writers may not make use of it or may make recommendations contrary to the findings of the review. Guideline writers should describe their search strategy and reasons for not including high quality evidence.

\section{P10 SURVEILLANCE OF HYPOCALCAEMIC SEIZURES SECONDARY TO VITAMIN D DEFICIENCY IN CHILDREN IN THE UK}

doi:10.1136/archdischild-2013-304107.010

E Basatemur, A Sutcliffe. General \& Adolescent Paediatrics Unit, UCL Institute of Child Health, London, UK

Background Reports suggest that rickets is an increasing concern among children in the UK, despite national recommendations for vitamin $\mathrm{D}$ supplementation during pregnancy and early childhood. However, there is limited epidemiological data to quantify these concerns, existing studies being limited to regional case series.

Methods Prospective national surveillance of hypocalcaemic seizures secondary to vitamin $D$ deficiency in children aged $0-15$ years, across the UK and Ireland via the British Paediatric Surveillance Unit (BPSU) system. We report results for the first 13 months of surveillance (September 2011 to September 2012).

Results 70 case notifications were received; 44 were confirmed cases, 6 probable cases, 17 reported in error or duplicates, and 3 unconfirmed cases for which details are pending. $90 \%$ of the 50 confirmed and probable cases were infants $(n=45)$, with three cases aged 1 year (6\%) and two aged 14 years (4\%). This equates to an incidence of 5.2 per 100,000 in infants. There was a male predominance of $76 \%(n=38)$. Ethnic distribution of cases is shown in the figure 1 . $60 \%$ of children $(n=30)$ had multiple seizures, and $24 \%$ $(n=11)$ had seizures lasting $>10$ minutes. $66 \%$ of cases did not exhibit any other clinical features of vitamin D deficiency $(n=33)$, $26 \%$ had clinical rickets $(n=13)$, and $8 \%$ had failure to thrive $(n=4)$. None of the children had fractures or intracranial haemorrhage. I.v. calcium gluconate was given in $48 \%$ of cases $(n=24)$ and anti-seizure medication in $26 \%(n=13)$, with $42 \%(n=21)$ not receiving any acute treatment. There were no deaths, and only one child had sequelae on discharge; an extravasation burn from i.v. calcium gluconate.

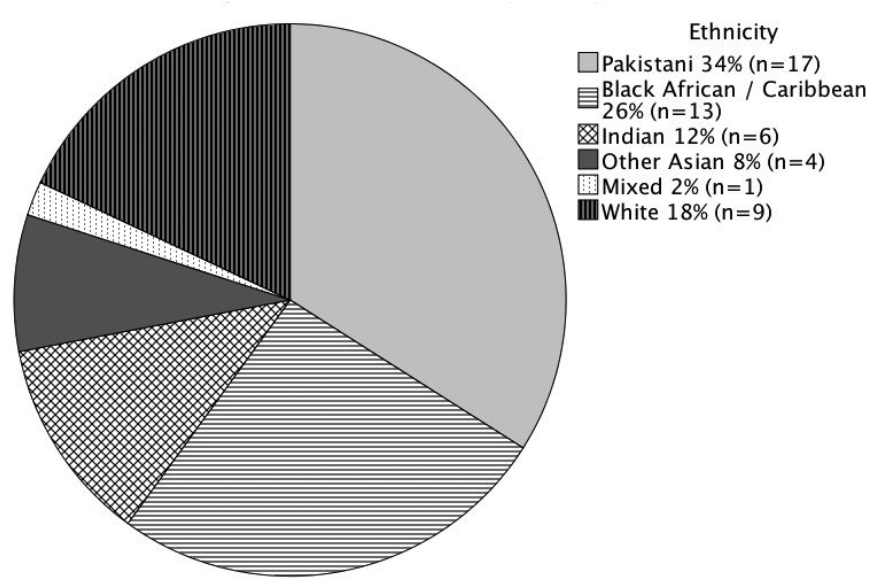

Abstract P10 Figure 1 Distribution of cases by ethnicity

Conclusions Although a relatively uncommon presentation of vitamin D deficiency, between September 2011 to September 2012 approximately one child has a seizure secondary to vitamin D deficiency per week in the UK. This suggests that current implementation of public health policy is not successful at preventing complications of severe vitamin D deficiency in children. Further studies are required to investigate the epidemiology of rickets more broadly in the UK.

\section{P11 CAN REAL-TIME PERFORMANCE FEEDBACK IMPROVE CHEST COMPRESSION QUALITY DURING SIMULATED INFANT CPR? A RANDOMISED CONTROLLED TRIAL}

doi:10.1136/archdischild-2013-304107.011

1PS Martin, 'PS Theobald, ${ }^{2} \mathrm{AM}$ Kemp, ${ }^{2} \mathrm{SA}$ Maguire, ${ }^{3} \mathrm{~K}$ Maconochie, 'MD Jones. ${ }^{1}$ Institute of Medical Engineering \& Medical Physics, Cardiff University, Cardiff, UK; IInstitute of Primary Care \& Public Health, Cardiff University, Cardiff, UK; ${ }^{3}$ Paediatric Emergency Department, St Mary's Hospital, London, UK

Aims Current International Liaison Committee on Resuscitation (ILCOR) guidelines emphasise the provision of high quality chest compressions during infant cardiopulmonary resuscitation (CPR). Recent research, however, reports that $<1 \%$ of all chest compressions achieve all four internationally recommended quality targets during simulated infant CPR. This study aimed to determine if 'real-time performance feedback' improved the quality of chest compressions provided during simulated infant CPR.

Methods Sixty-nine certified European and Advanced Paediatric Life Support (EPLS and APLS) training course instructors were recruited from seven EPLS/APLS training courses. Instructors were randomly 